\title{
Propagation of spatial coherence in fast pulses
}

\author{
Robert W. Schoonover, ${ }^{1, *}$ Brynmor J. Davis, ${ }^{1}$ Randy A. Bartels, ${ }^{2}$ and P. Scott Carney ${ }^{1}$ \\ ${ }^{1}$ Department of Electrical and Computer Engineering and The Beckman Institute for Advanced Science and \\ Technology, University of Illinois at Urbana-Champaign, 405 N. Mathews, Urbana, Illinois 61801, USA \\ ${ }^{2}$ Department of Electrical and Computer Engineering, Colorado State University, Fort Collins, Colorado 80523, USA \\ ${ }^{*}$ Corresponding author: rschoono@illinois.edu
}

Received April 16, 2009; accepted June 18, 2009; posted July 9, 2009 (Doc. ID 110140); published August 14, 2009

Diffraction and interferometry with fast pulses are analyzed for the case that the fields are partially correlated in time and in space. This generalizes a previous work [Schoonover et al., J. Mod. Opt. 55, 1541 (2008)], where only the temporal correlations of pulsed fields were considered in a Young's interferometer. The meaning of the interferograms is addressed for measurements taken in the near, Fresnel, and far zones of the source. It is shown that single-shot measurements cannot generally be used to infer statistical properties of the source, rather, data averaged over many pulses must be used. (C) 2009 Optical Society of America

OCIS codes: 260.1960, 030.0030, 320.7120.

\section{INTRODUCTION}

Statistical optics and coherence theory [1,2] provide a means to interpret and predict the results of experiments involving random fields. Those random optical fields are often taken to be statistically stationary, at least in the wide sense. The assumptions of stationarity and ergodicity allow time-averaged quantities to be related to ensemble-averaged quantities and, using the WienerKhintchine-Einstein theorem, allow the temporal autocorrelation of the field to be related to the power spectrum of the field [3-5]. When the assumption of stationarity does not hold, the correlation functions that describe the field become two-time or two-frequency correlation functions, and the Wiener-Khintchine-Einstein theorem no longer holds. For these reasons and others, the timeaveraged measurements made with an interferometer or a spectrometer have a more complicated relationship with the nonstationary sources that generated them [6-8] than in the stationary case. Some sort of prior knowledge is necessary to make unambiguous estimates of the statistical properties of the sources based on the measured data.

While stationary fields are not strictly realizable, e.g.,. they are random processes without beginning or end, the stationary model often well describes measurements. However, recent advances in short pulse generation have moved the frontier of statistical optics into a regime where the stationary model clearly fails. Ultrashort pulses and high-repetition-rate lasers are increasingly common, and their use in metrology is becoming ubiquitous $[9,10]$. By definition, a pulsed source is nonstationary. As the use of such devices grows, so to does the need to understand measurements using nonstationary theory.

In recent experiments, attempts have been made to measure the coherence properties of pulsed optical systems, including estimating coherence properties [11,12], the degree of spatial coherence of a beam [13-18], and the power spectrum $[8,19]$. However, the interpretation of these results is based either on the theory of stationary random processes or on a completely general theory of nonstationary fields ([8] being an exception in which cyclostationarity is invoked). It is not clear that notions of coherence or power spectra even apply in some such experiments and where they do, it is in a generalized sense.

Generalized spectra and cross-spectral densities for a number of classes of nonstationary fields have been defined and investigated $[6,20,21]$. In many of the experiments described above, the field is made up of a large number of stochastic pulses with a periodic probability density function exhibiting a fixed temporal period. The random field may then be well modeled as a cyclostationary random process [22-24]. While accommodating pulses, such a model retains many of the advantages of the more restrictive theory of stationary random processes. In particular, if the fields are also cycloergodic, certain time averages may be exchanged for the appropriate ensemble averages; spectra and cross-spectra emerge from clear generalizations of the Wiener-KhintchineEinstein theorem, and these quantities may be related to the observable, physical spectra [24,25].

It is clear that real experiments are conducted with fields of finite temporal support, which are to some degree stochastic with varying fluctuations at different points in space. The methods used to generate fast pulses, e.g., high harmonic generation [13,26], may also produce differing fluctuations at distinct locations in the source. For this reason, a spatiotemporal stochastic model is developed here. Within the context of the stationary, ergodic theory, such fields are commonly called partially spatially coherent. In order to provide a framework of analysis for experiments involving pulsed stochastic fields, a particular model, not completely general, for pulsed, partially spatially coherent fields is presented and explored. Specifically, a partially spatially coherent stationary field is deterministically temporally modulated. The mathematical framework presented is used to relate measurable quantites to properties of the source. It is shown that the interference pattern created in a Young's experiment depends on the deterministic properties of the modulation 
that determined the pulse shape, chirp, duration, and pulse repetition period; the distance between the source and the Young's interferometer; and the statistical properties of the underlying stationary source.

This paper is organized as follows: in the remainder of Section 1, the basic theory of cyclostationary optical fields is described. In Section 2, the propagation of fields in free space is described, and measurements of the field are discussed in the context of interferometry. In Section 3, simulations illustrate the results. These simulations include interferometric measurements made in different regions of space away from the source. Potential uses of these interferometric techniques in elucidating statistical properties of the original source are discussed. Conclusions are drawn in Section 4.

The pulsed, stochastic fields considered in this paper arise from a stationary optical source that is allowed to radiate at discrete, well-described intervals by some external means, such as a shutter. The term shutter is used throughout this paper to generically describe the mechanism of shaping the pulse. The resulting field forms a pulse train with a fixed time between pulses. This model encompasses only a small subset of possible cyclostationary fields, but may model the field well for certain methods of short pulse generation. For example, in high harmonic generation, a laser pulse is incident on an atomic gas, which then emits at harmonics of the impinging laser frequency. The collection of atoms serve as the stochastic source, and the impinging laser field acts as a kind of modulation. Likewise, optical pulses produced by Q-switching [27] may be considered cyclostationary when the modulation of the loss in the cavity is periodic. Timing jitter, which has been treated elsewhere [28], can be cast as a cyclostationary phenomena, although it does not fit into the intrinsically stationary, cyclostationary model in this paper. To provide a context for analysis, the secondorder moments of the field, the mutual coherence function, the cross-spectral density, and the generalized crossspectral density are developed below.

A stochastic, statistically stationary, planar, secondary source has the mutual coherence function

$$
\bar{\Gamma}_{P}\left(\boldsymbol{\rho}_{1}, \boldsymbol{\rho}_{2}, \tau\right)=\left\langle\bar{U}^{*}\left(\boldsymbol{\rho}_{1}, t-\tau\right) \bar{U}\left(\boldsymbol{\rho}_{2}, t\right)\right\rangle,
$$

where $\bar{U}(\boldsymbol{\rho}, t)$ is the random field at position $\boldsymbol{\rho}$ and time $t$ and the angle brackets denote an ensemble average over the fluctuating scalar field. Stationarity ensures that the coherence function is not dependent on the time $t$. The field is assumed to be ergodic, so the ensemble average is the same as the long-time average. This source is assumed to be modulated in time such that the resulting field is no longer stationary. The two-time correlation function, $\Gamma_{P}$, of the nonstationary field on a plane (usually the exit plane of the optical system) is related to the stationary mutual coherence function by the expression

$$
\begin{aligned}
\Gamma_{P}\left(\boldsymbol{\rho}_{1}, \boldsymbol{\rho}_{2}, t-\tau, t\right) & =\left\langle U^{*}\left(\boldsymbol{\rho}_{1}, t-\tau\right) U\left(\boldsymbol{\rho}_{2}, t\right)\right\rangle \\
& =\bar{\Gamma}_{P}\left(\boldsymbol{\rho}_{1}, \boldsymbol{\rho}_{2}, \tau\right) h^{*}(t-\tau) h(t),
\end{aligned}
$$

where $U(\boldsymbol{\rho}, t)=\bar{U}(\boldsymbol{\rho}, t) h(t), h(t)$ is a deterministic modulation function that describes the modulation of the source and the angle brackets denote an average over the en- semble of the underlying stationary process as in Eq. (1). The overbar denotes quantities associated with the underlying stationary field.

Suppose the modulation function is periodic, thus having the representation

$$
h(t)=\sum_{n} h_{n} e^{-i \omega_{0} n t}, \quad \omega_{0}=\frac{2 \pi}{T_{0}},
$$

where $\left\{h_{n}\right\}$ are complex coefficients and $T_{0}$ is the repetition time of the system. It is possible to choose the modulation $h(t)$ to incorporate the pulse shape, chirp, etc. This modulation function, when multiplied by the field at the source, yields a source that is cyclostationary. Cyclostationary random processes exhibit a discrete time translation symmetry, $\Gamma\left(\boldsymbol{\rho}_{1}, \boldsymbol{\rho}_{2}, t-\tau, t\right)=\Gamma\left(\boldsymbol{\rho}_{1}, \boldsymbol{\rho}_{2}, t-\tau+T_{0}, t+T_{0}\right)$ for some $T_{0}$, the cyclostationary period. This is in contrast to stationary random processes, in which the time translation symmetry holds for any value of $T_{0}$. In many practical cases, cycloergodicity can be invoked to give a relation between measurements and field statistics.

Since the underlying source is assumed to be stationary, the cross-spectal density, $W$, and the mutual coherence function, $\Gamma$, are simply related by a Fourier transform,

$$
\bar{W}_{P}\left(\boldsymbol{\rho}_{1}, \boldsymbol{\rho}_{2}, \omega\right)=\int_{-\infty}^{\infty} \mathrm{d} \tau \bar{\Gamma}_{P}\left(\boldsymbol{\rho}_{1}, \boldsymbol{\rho}_{2}, \tau\right) e^{i \omega \tau}
$$

The two-frequency cross-spectral density for the modulated field is given by the two-time Fourier transform of the two-time mutual coherence function [1],

$$
\begin{aligned}
& W_{P}\left(\boldsymbol{\rho}_{1}, \boldsymbol{\rho}_{2}, \omega, \omega+\Omega\right) \\
& \quad=\iint \mathrm{d} t \mathrm{~d} \tau \Gamma_{P}\left(\boldsymbol{\rho}_{1}, \boldsymbol{\rho}_{2}, t-\tau, t\right) e^{i(\omega \tau+\Omega t)} \\
& \quad=\sum_{m, n} h_{n}^{*} h_{m+n} \bar{W}\left(\boldsymbol{\rho}_{1}, \boldsymbol{\rho}_{2}, \omega-\omega_{0} n\right) \delta\left(\Omega-m \omega_{0}\right),
\end{aligned}
$$

where, again, the overbar denotes the cross-spectral density of the underlying stationary source. The twofrequency correlations are nonzero only when the frequencies differ by multiples of the repetition frequency of the system, a generalization of the Wiener-KhintchineEinstein theorem [21].

For fields of the type described in this paper, there are three important time scales: the coherence time of the underlying source, $\tau_{c}$; the duration of a single pulse, $T$; and the pulse repetition period, $T_{0}$. These values all affect the statistical properties of the cyclostationary field. Based on the relative scales of these three quantities, there are three qualitatively distinct regimes into which most realizable systems fall [7]:

- Regime I is defined by $T<T_{0} \ll \tau_{c}$. Pulses separated by several $T_{0}$ exhibit significant correlations in this regime.

- Regime II is defined by $T<\tau_{c}<T_{0}$. Pulses separated by the pulse period $T_{0}$ may exhibit a significant statistical relationship, but pulses separated by multiple repetition periods are statistically uncorrelated. 
- Regime III is defined by $\tau_{c} \ll T<T_{0}$, In this limit, the pulses are separated by significantly more than $\tau_{c}$ and so separate pulses are not statistically related. Additionally, the condition $\tau_{c} \ll T$ indicates that the field at the beginning of any pulse is uncorrelated with the field at the end of that pulse.

\section{PROPAGATION OF CORRELATIONS AND INTERFEROMETRY}

In works on nonstationary fields $[6,7,20]$, the emphasis has been on sources that are spatially fully coherent and of infinite extent. Such sources produce polychromatic plane waves. Real sources are of finite extent and can fluctuate independently at different points in space. An analysis of such partially spatially coherent sources and the fields they produce cannot be performed by analyzing a single polychromatic plane wave. Other works $[20,29]$ have dealt with spatial correlation functions of nonstationary fields within the context of coherent mode decompositions or the one-dimensional behavior of the twofrequency cross-spectral density function. Here, the propagation of partially spatially coherent cyclostationary statistical quantities is addressed.

A random field can be characterized by the ensemble of possible realizations of that field. For optical fields, each member of the ensemble obeys the wave equation (Helmholtz equation). Each member of the ensemble may then be propagated individually using the standard method of Green functions. In the time domain, the propagated field is

$$
U_{S}(\mathbf{r}, t)=\int \mathrm{d} t^{\prime} \mathrm{d}^{3} r^{\prime} G\left(\mathbf{r}, \mathbf{r}^{\prime}, t, t^{\prime}\right) U_{P}\left(\mathbf{r}^{\prime}, t^{\prime}\right)+\text { b.c. },
$$

where the causal Green function in free space is

$$
G\left(\mathbf{r}, \mathbf{r}^{\prime}, t, t^{\prime}\right)=\frac{\delta\left(t-t^{\prime}-\frac{\left|\mathbf{r}-\mathbf{r}^{\prime}\right|}{c}\right)}{\left|\mathbf{r}-\mathbf{r}^{\prime}\right|},
$$

$U_{P}$ is a member of the ensemble of the source distribution, $U_{S}$ is the corresponding member of the ensemble propagated from the source, $c$ is the speed of light, and b.c. stands for the terms resulting from satisfying boundary conditions. In the frequency domain, the propagated field is

$$
\widetilde{U}_{S}(\mathbf{r}, \omega)=\int \mathrm{d}^{3} r^{\prime} \widetilde{G}\left(\mathbf{r}, \mathbf{r}^{\prime} ; k\right) \widetilde{U}_{P}\left(\mathbf{r}^{\prime}, \omega\right)+\text { b.c. }
$$

where the spectral Green function in free space is

$$
\widetilde{G}\left(\mathbf{r}, \mathbf{r}^{\prime} ; k\right)=\frac{e^{i k\left|\mathbf{r}-\mathbf{r}^{\prime}\right|}}{\left|\mathbf{r}-\mathbf{r}^{\prime}\right|},
$$

and $k=\omega / c$. The tilde denotes a Fourier transform on the temporal variable. After propagating the fields, the average over the members of the ensemble can then be taken to obtain the resultant mutual coherence function (crossspectral density) for the propagated field. The terms re- sulting from boundary conditions are taken to be zero for the rest of this paper.

The averaging process may be carried out before propagation, in which case the Green functions are given by

$$
K\left(\mathbf{r}_{1}, \mathbf{r}_{1}^{\prime}, \mathbf{r}_{2}, \mathbf{r}_{2}^{\prime}, t_{1}, t_{1}^{\prime}, t_{2}, t_{2}^{\prime}\right)=G^{*}\left(\mathbf{r}_{1}, \mathbf{r}_{1}^{\prime}, t_{1}, t_{1}^{\prime}\right) G\left(\mathbf{r}_{2}, \mathbf{r}_{2}^{\prime}, t_{2}, t_{2}^{\prime}\right),
$$

$$
\widetilde{K}\left(\mathbf{r}_{1}, \mathbf{r}_{1}^{\prime}, \mathbf{r}_{2}, \mathbf{r}_{2}^{\prime} ; k_{1}, k_{2}\right)=\widetilde{G}^{*}\left(\mathbf{r}_{1}, \mathbf{r}_{1}^{\prime} ; k_{1}\right) \widetilde{G}\left(\mathbf{r}_{2}, \mathbf{r}_{2}^{\prime} ; k_{2}\right) .
$$

The propagated second-order coherence functions are then given by the integrals

$$
\begin{aligned}
\Gamma_{S}\left(\mathbf{r}_{1}, \mathbf{r}_{2}, t_{1}, t_{2}\right)= & \int \mathrm{d} t_{1}^{\prime} \mathrm{d} t_{2}^{\prime} \mathrm{d}^{3} r_{1}^{\prime} \mathrm{d}^{3} r_{2}^{\prime} K\left(\mathbf{r}_{1}, \mathbf{r}_{1}^{\prime}, \mathbf{r}_{2}, \mathbf{r}_{2}^{\prime}, t_{1}, t_{1}^{\prime}, t_{2}, t_{2}^{\prime}\right) \\
& \times \Gamma_{P}\left(\mathbf{r}_{1}^{\prime}, \mathbf{r}_{2}^{\prime}, t_{1}^{\prime}, t_{2}^{e}\right)
\end{aligned}
$$

and

$$
\begin{aligned}
W_{S}\left(\mathbf{r}_{1}, \mathbf{r}_{2}, \omega_{1}, \omega_{2}\right)= & \int \mathrm{d}^{3} r_{1}^{\prime} \mathrm{d}^{3} r_{2}^{\prime} \tilde{K}\left(\mathbf{r}_{1}, \mathbf{r}_{1}^{\prime}, \mathbf{r}_{2}, \mathbf{r}_{2}^{\prime}, k_{1}, k_{2}\right) \\
& \times W_{P}\left(\mathbf{r}_{1}^{\prime}, \mathbf{r}_{2}^{\prime}, \omega_{1}, \omega_{2}\right) .
\end{aligned}
$$

The field, or moments and correlations of the field may be measured with some form of interferometry. Young's classic interference experiment [30] is used even today to measure two-point correlations of optical fields ([31], see Chap. 15 and references therein). The utility of such an experiment springs from the fact that, for stationary fields, the visibility of the interferogram is equal to the magnitude of the spatial degree of coherence of the field at the two apertures. The interferogram produced in such an experiment also can be used to determine the approximate coherence time (and thus the bandwidth) of the impinging field. For cyclostationary plane waves, it has been shown that in a Young's interferometer the meaning of the interferogram is changed and there is some ambiguity in connecting the results to the source parameters [7]. Specifically, it was shown that the smaller of the pulse duration, $T$, and the coherence time, $\tau_{c}$, sets the width of the primary peak in the interferograms. The extent to which spatial correlation properties of the field, or the source that generated the field, may be investigated through interferometric measurements has not been established elsewhere and is addressed below.

The Young's two-pinhole interferometer, as shown in Fig. 1, contains a screen $\mathcal{A}$ that includes two pinhole apertures and a detector in the plane $\mathcal{D}$, parallel to $\mathcal{A}$. The screen $\mathcal{A}$ is chosen to be parallel to the source plane, here defined by the shutter, and is a distance $z^{\prime}$ away from the source.

For points $P$ on the detection screen, the ensemble average of the instantaneous intensity as a function of time is related to the fields in the two pinholes by the equation 


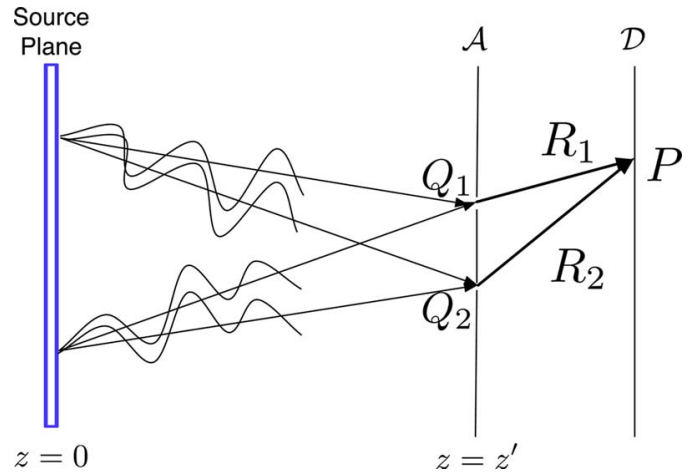

Fig. 1. (Color online) Diagrammatic sketch of the source and interferometer.

$$
\begin{aligned}
\Gamma_{\mathcal{D}}(P, P, t, t)= & \left|\kappa_{1}\right|^{2} \Gamma_{\mathcal{A}}\left(Q_{1}, Q_{1}, t-R_{1} / c, t-R_{1} / c\right) \\
& +\left|\kappa_{2}\right|^{2} \Gamma_{\mathcal{A}}\left(Q_{2}, Q_{2}, t-R_{2} / c, t-R_{2} / c\right) \\
& +\kappa_{1}^{*} \kappa_{2} \Gamma_{\mathcal{A}}\left(Q_{1}, Q_{2}, t-R_{1} / c, t-R_{2} / c\right) \\
& +\kappa_{1} \kappa_{2}^{*} \Gamma_{\mathcal{A}}\left(Q_{2}, Q_{1}, t-R_{2} / c, t-R_{1} / c\right),
\end{aligned}
$$

where $\Gamma_{\mathcal{A}}$ is the mutual coherence function at plane $\mathcal{A}$, the $\kappa_{i}$ are constant (for observation points close to the optical axis) factors that depend on the area of the pinholes and the distance $R_{i}$ from the $i$ th pinhole to the point $P$ (see Fig. 1). For fields that are quasi-monochromatic, $\kappa_{i}=$ $-i \mathrm{~d} \mathcal{A} / \bar{\lambda} R_{i}$ [32], where $\bar{\lambda}=2 \pi c / \omega_{c}$ and $\mathrm{d} \mathcal{A}$ is the area of the pinhole. Using Eq. (14), the interferogram created by a field with a known cross-spectral density at the pinholes may be computed.

The visibility in an interferometric measurement [1] is

$$
\mathcal{V}=\frac{I_{\max }-I_{\min }}{I_{\max }+I_{\min }} .
$$

For stationary fields, when the intensity in each pinhole is approximately the same, the visibility is related to the complex degree of coherence, $\gamma$, via $\mathcal{V}=\mid \gamma\left[Q_{1}, Q_{2},\left(R_{2}\right.\right.$ $\left.\left.-R_{1}\right) / c\right] \mid, \quad$ where $\quad \gamma\left(Q_{1}, Q_{2}, \tau\right)=\Gamma\left(Q_{1}, Q_{2}, \tau\right) /$ $\sqrt{\Gamma\left(Q_{1}, Q_{1}, 0\right) \Gamma\left(Q_{2}, Q_{2}, 0\right)}$ is a normalized measure of statistical similarity between two points. The visibility ranges from zero to unity as the field goes from incoherent to fully coherent.

\section{SIMULATIONS}

Numerical simulations were performed to illustrate the observable effects of cyclostationarity and their connection to the parameters of the source in pulsed, spatially partially coherent fields. Closely following the method outlined in [33], realizations of a discrete random process were generated to model the stationary source, which is assumed to be planar. For all of the simulations in this manuscript, the modulation function was taken to be square waves with duration $T$ and duty cycle $T / T_{0}$. The correlation function of the field at the shutter was assumed to be factorizable in time and space, viz.,

$$
\bar{\Gamma}\left(\mathbf{r}_{1}, \mathbf{r}_{2}, \tau\right)=\Gamma^{\prime}(\tau) F\left(\mathbf{r}_{1}, \mathbf{r}_{2}\right),
$$

where $F$ is a function of the two position coordinates $\mathbf{r}_{1}$ and $\mathbf{r}_{2}$ that lie in the shutter plane and expresses the spatial correlations of the source and the amplitudes across the source plane. For the simulations, $\Gamma$ and $F$ were taken to be Gaussian:

$$
\begin{gathered}
\Gamma^{\prime}(\tau)=\exp \left(-\tau^{2} / 2 \tau_{c}^{2}\right) \exp \left(-i \omega_{c} \tau\right), \\
F\left(\mathbf{r}_{1}, \mathbf{r}_{2}\right)=\exp \left(-r_{1}^{2} / 4 \sigma_{s}^{2}\right) \exp \left(-r_{2}^{2} / 4 \sigma_{s}^{2}\right) \exp \left(-\left|\mathbf{r}_{1}-\mathbf{r}_{2}\right|^{2} / 2 \sigma_{g}^{2}\right),
\end{gathered}
$$

where $\sigma_{s}$ and $\sigma_{g}$ are the beam width and coherence length at the secondary source, respectively, $\omega_{c}$ is the central frequency, and $\tau_{c}$ is the coherence time. For all simulations, except where noted, the central frequency is $\omega_{c}=1$ $\times 10^{15} \mathrm{rad} / \mathrm{s} \quad\left(\lambda_{c}=1.88 \mu \mathrm{m}\right)$, the interferometer has pinhole separation $s=2 \mathrm{~mm}$ and the distance between the screen and the detection plane is $d=50 \mathrm{~mm}$. The beam width and coherence lengths were chosen throughout this paper to ensure that the resultant field is always beamlike, that is, both of the length parameters, $\sigma_{s}$ and $\sigma_{g}$, are much larger than the largest wavelength for which the cross-spectral density is not negligible. The source plane was discretized, and an independent zero-mean, complex Gaussian random variable was generated for each spatial point for each time step in the simulation. A twodimensional spatial filter was applied to the complex Gaussian random variable for each time step to give the chosen spatial coherence properties, while a onedimensional temporal filter was applied at each position in the discretized source to give the chosen temporal coherence properties. The random process at each point source was then weighted to give the chosen field amplitude. The coherence properties and amplitude used in these simulations are defined in Eqs. (16)-(18).

Following the simulation of the source in the plane, the temporal modulation, defined by $h$, was applied to the source, and the field was propagated using the causal Green function [see Eq. (6)], treating each point in the plane as a primary point source. The total field at each pinhole, assumed infinitely small, was calculated by summing the contributions from each source point. It was assumed that the detector was frequency independent, i.e., that the measured intensity in the detection plane was the square magnitude of the sum of the two incident fields. Long-time averages were taken of the instantaneous intensity in the detection plane and plotted for a variety of source distributions.

Simulations were run for sources in the different temporal regimes described above, with different spatial coherence and intensity profiles. The resultant fields generated by such sources were then propagated numerically, using Eq. (6), to the two pinholes, and the resultant interferograms were calculated. In these simulations, a Young's interferometer (i.e., a planar screen with two pinholes, $\mathcal{A}$, and a detection plane, $\mathcal{D}$, as seen in Fig. 1 ) was placed at three different distances from the source-one near the source, one in an intermediate (Fresnel) zone, and one in the far zone-to help discern different propagation effects. 


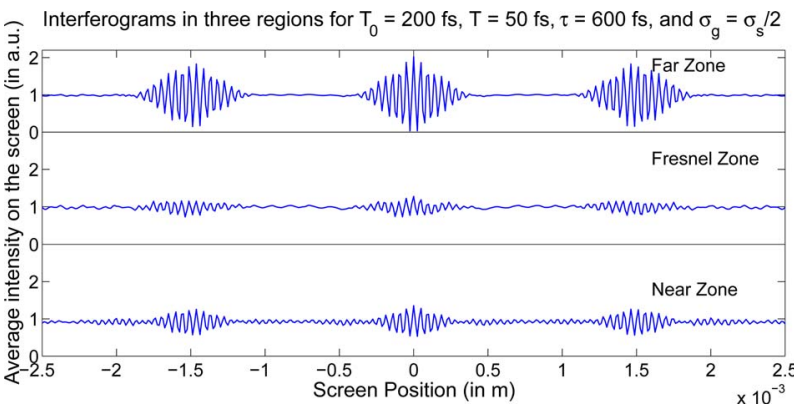

Fig. 2. (Color online) Simulated interferograms for a source with $T_{0}=200 \mathrm{fs}, T=50 \mathrm{fs}, \tau_{c}=600 \mathrm{fs}$, and $\sigma_{s}=\sigma_{s} / 2=1 \mathrm{~mm}$. The top panel contains the interferogram as would be measured in the far zone (100 m away from the source), the middle panel contains the interferogram as would be measured in the Fresnel zone (500 $\mathrm{mm}$ away from the source), and the bottom panel contains the interferogram as would be measured in the near zone $(2 \mathrm{~mm}$ away from the source). This source is in Regime I.

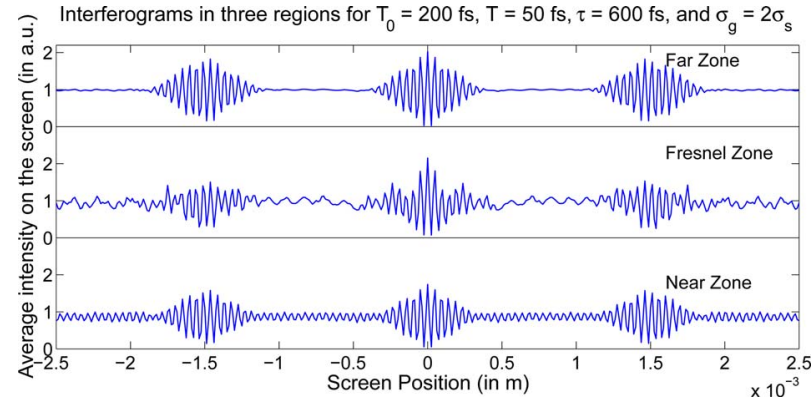

Fig. 3. (Color online) Simulated interferograms for a source with $T_{0}=200 \mathrm{fs}, T=50 \mathrm{fs}, \tau_{c}=600 \mathrm{fs}$, and $\sigma_{g}=2 \sigma_{s}=4 \mathrm{~mm}$. Panel descriptions as for Fig. 2. This source is in Regime I.

In Figs. 2-7, 18 interferograms are shown for a source characterized by three time scales (the pulse duration, $T$; the repetition period, $T_{0}$; and the coherence time, $\tau_{c}$ ), two sets of spatial parameters (the coherence length, $\sigma_{g}$; and the beam width in the plane, $\sigma_{s}$ ), and in three separate detection regions (zones). A change in zone denotes a change in the distance between the shutter and the plane $\mathcal{A}$ in Fig. 1. It may be noted that as the spatial coherence of the source is increased, the visibility of the fringes increases as measured in both the Fresnel and near zones. In the far zone, the interferograms appear to have unit visibility, regardless of the spatial coherence properties of the source. This is because propagation to the far zone produces a spatially coherent field across the pinholes.

Far zone. Much like in the theory of stationary fields,

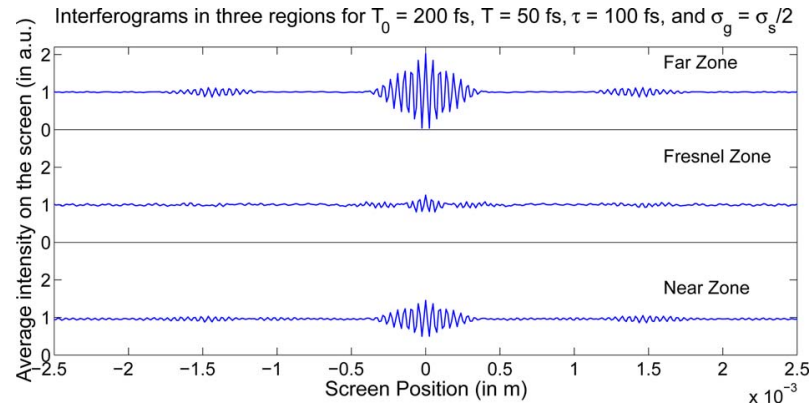

Fig. 4. (Color online) Simulated interferograms for a source with $T_{0}=200 \mathrm{fs}, T=50 \mathrm{fs}, \tau_{c}=100 \mathrm{fs}$, and $\sigma_{g}=\sigma_{s} / 2=1 \mathrm{~mm}$. Panel descriptions as for Fig. 2. This source is in Regime II.

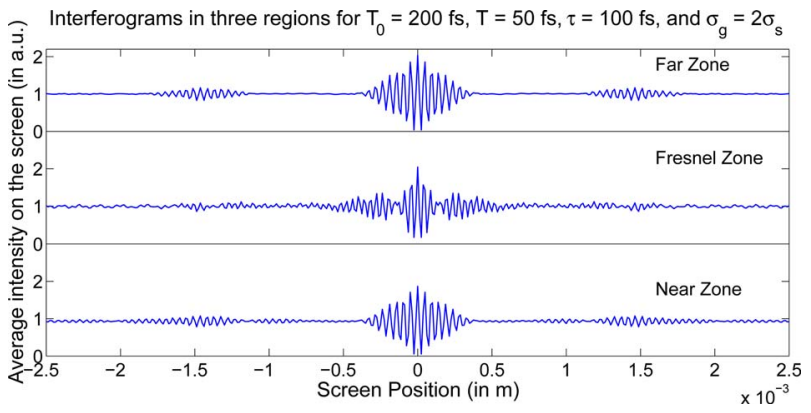

Fig. 5. (Color online) Simulated interferograms for a source with $T_{0}=200 \mathrm{fs}, T=50 \mathrm{fs}, \tau_{c}=100 \mathrm{fs}$, and $\sigma_{g}=2 \sigma_{s}=4 \mathrm{~mm}$. Panel descriptions as for Fig. 2. This source is consistent with being in Regime II.

the cross-spectral density in the far zone is related to the Fourier transform of the source cross-spectral density function $F$. The far zone is defined as any distance in which the source appears pointlike [[32], see Eq. (33) in Sec. 8.3], or when

$$
\frac{z_{0}}{a} \gg \frac{a}{4 \lambda}
$$

where $z_{0}$ is the distance between the object and interferometer, $a$ is the cross section of the source, and $\lambda$ is the wavelength. The spectral Green function in Eq. (9) can be replaced by $\widetilde{G}\left(\mathbf{r}, \mathbf{r}^{\prime} ; k\right) \approx \exp (i k r) \exp \left(-i k \hat{r} \cdot \mathbf{r}^{\prime}\right) / r$ when Eq. (19) applies. For the specific geometry described in this paper, the field incident on the two pinholes in the far zone is indistinguishable from a single polychromatic plane wave traveling in the $\hat{z}$ direction with cross-spectral density

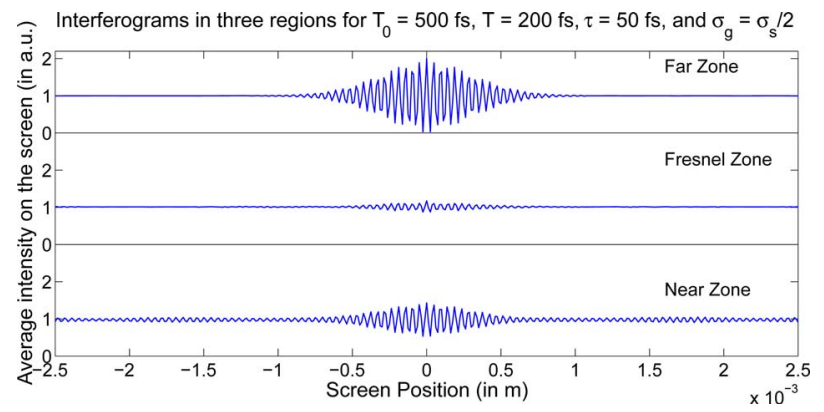

Fig. 6. (Color online) Simulated interferograms for a source with $T_{0}=500 \mathrm{fs}, T=200 \mathrm{fs}, \tau_{c}=50 \mathrm{fs}$, and $\sigma_{g}=\sigma_{s} / 2=1 \mathrm{~mm}$. Panel descriptions as for Fig. 2. This source is in Regime III.

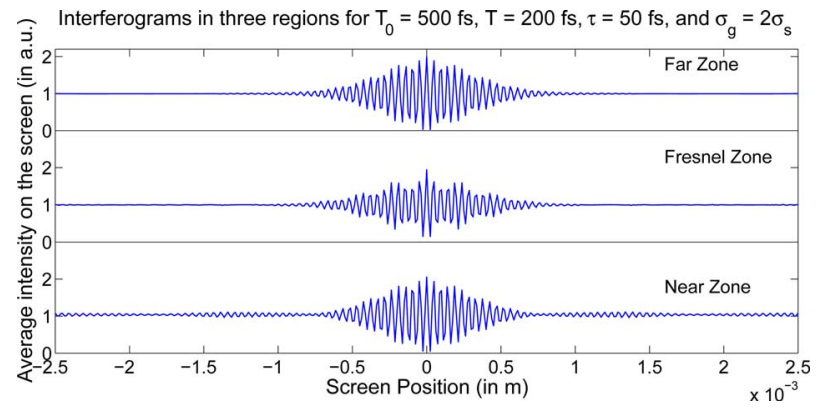

Fig. 7. (Color online) Simulated interferograms for a source with $T_{0}=500 \mathrm{fs}, T=200 \mathrm{fs}, \tau_{c}=50 \mathrm{fs}$, and $\sigma_{g}=2 \sigma_{s}=4 \mathrm{~mm}$. Panel descriptions as for Fig. 2. This source is in Regime III. 


$$
W^{(\infty)}(\omega, \omega+\Omega)=\widetilde{F}(\mathbf{0}, \mathbf{0}) A(\omega, \omega+\Omega) e^{i \Omega r} / r^{2},
$$

where $\widetilde{F}$ is the four-dimensional Fourier transform of $F$, $A(\omega, \omega+\Omega)$ is the two-frequency cross spectral density of the modulated source [found by taking the double Fourier transform of $\left.\Gamma^{\prime}\left(t_{2}-t_{1}\right) h^{*}\left(t_{1}\right) h\left(t_{2}\right)\right]$, and $W^{(\infty)}$ is position independent for small pinhole spacings, $s$. Thus, for sources that are well described by Eq. (16), only the temporal correlation and modulations, described by the function $A$, may be investigated. The spatial correlations of the source are manifest only in the constant multiplier $\widetilde{F}(\mathbf{0}, \mathbf{0})$. Some of the temporal coherence or modulation properties of the source may be determined or certain parameters of it may be estimated. The width of the primary interference peak is determined by the smaller of $T$ or $\tau_{c}$ [7]. The distance between the primary peak and any secondary peaks (as seen in Figs. 2 and 3) maps to the cyclostationary period, $T_{0}$ [7]. It is noted that in the results of the simulations shown in the top panels of Figs. 2-7, the visibility is nearly unity, as should be for the case of a plane wave incident perpendicular to the plane of the twopinhole interferometer. The minor variations from unity are a result of the very minor variations of the angular positions of the two pinholes viewed from the source plane.

Fresnel zone. The Fresnel zone is an intermediate region away from the source and near the beam axis. In this region, the spectral Green function, Eq. (9), takes the form $\widetilde{G}\left(\mathbf{r}, \mathbf{r}^{\prime} ; k\right) \approx \exp (i k z) / z \exp \left[i k\left(\boldsymbol{\rho}-\boldsymbol{\rho}^{\prime}\right)^{2} /(2 z)\right]$. In this zone, the radiated field does not appear as a single polychromatic plane wave as in the far zone. The field radiated from different points in the source plane may have differences in times of flight to the pinholes of up to $15 \mathrm{fs}$ for the parameters in these simulations. The fields radiated from these points, though, are only partially correlated. Depending on the coherence length at the source, the contributions to the field at the pinholes from these points may, upon averaging, interfere, creating an interferogram that has a central peak wider than is found in the far zone. For example, in Fig. 5, the major peak in the interferogram as simulated in the Fresnel zone is wider than the peaks in the other two zones whereas in Fig. 6, the interference effects essentially wash out all the fringes in the Fresnel zone interferogram. In Fig. 8, the Fresnel-zone interferograms for three sources with identical temporal properties but different spatial coherence lengths are displayed. As the coherence length at the source increases, the field at the two pinholes more closely resembles the field that would be produced by a fully spatially coherent source. Note that the central peak becomes wider and more visible as the coherence length at the source increases.

Near zone. In the near zone, both the amplitude rolloff of $1 / r$ and the varying phase accumulations between all source points and the pinholes are important; thus the propagator $\widetilde{G}$ cannot be simplified. In Figs. 2-7, it is evident that the central peak has approximately the same width as in the far zone. This width is determined by the smaller of the pulse duration or coherence time. This is due to the fact that the interferometer is essentially sampling the field only at the points nearest the pinholes. The

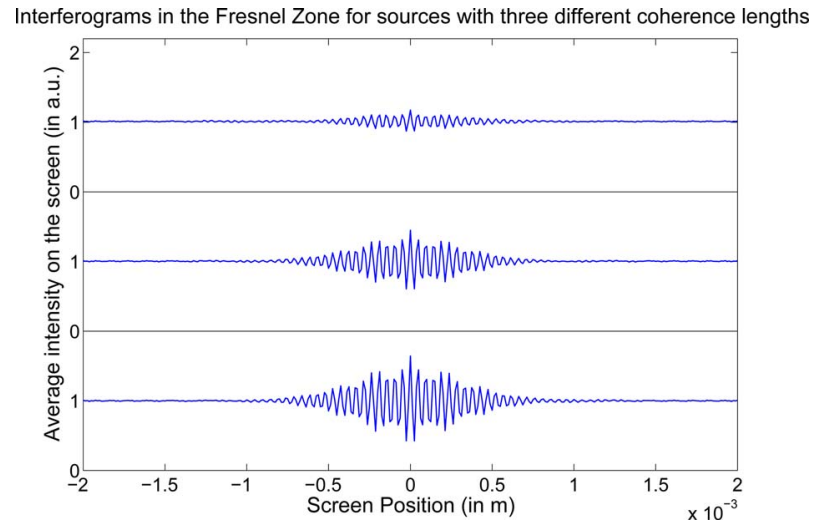

Fig. 8. (Color online) Simulated interferograms for three sources with $T_{0}=500 \mathrm{fs}, T=200 \mathrm{fs}, \tau_{c}=50 \mathrm{fs}$ and $\sigma_{s}=2 \mathrm{~mm}$. In the top panel, the simulated source has coherence length $\sigma_{g}=1 \mathrm{~mm}$, in the middle panel, the simulated source has coherence length $\sigma_{g}=1.5 \mathrm{~mm}$, and in the bottom panel, the simulated source has coherence length $\sigma_{g}=2 \mathrm{~mm}$.

duration of the pulse in the pinholes is then essentially the same as at the source. This is in contrast to the Fresnel zone, where the duration of the pulse has increased because of the varying times of flight to the pinholes from the extended source. The visibility of the main interferogram is an increasing function of the spatial coherence length at the source.

In both the near and intermediate zones, the visibility of the center fringe increases as the spatial coherence of the source increases. The far zone visibility measurements are, to a good approximation, independent of the spatial correlation properties of the source. In the Fresnel zone geometry, the field in each pinhole is dependent on the fields over a significant area of the source. The varying times of flight across this area cause the observed data to exhibit a multiplex dependence on the spatial and temporal statistics of the source. For a stationary source, variation of the statistics is independent of the origin of time. The nonstationary nature of the sources considered complicates the interpretation of the Fresnel zone data, as the detection-plane statistics are a function of the times of flight from each point in the source plane to the pinholes. Measurements in the near zone, however, may be sufficient for determining spatial correlation properties of the source, as will be seen in the next section.

\section{A. Determining Spatial Coherence Properties}

It is clear from the near zone analysis that no meaningful quantitative statements can be made about the spatial coherence properties of the source from measurements made in the far zone and that measurements made in the Fresnel zone may be useful only for qualitative statements about the spatial partial coherence. However, when the interferometer is placed near the source (for beamlike fields, within approximately the width of the intensity in the source plane), there is a relationship between the visibility in the interferograms and the complex degree of spatial coherence of the underlying stationary process when the stationary process is factorizable, as in in Eq. (16).

In Fig. 9, the near zone visibility is plotted against the magnitude of the complex degree of coherence between 
The measured visibility versus the magnitude of the complex degree of coherence

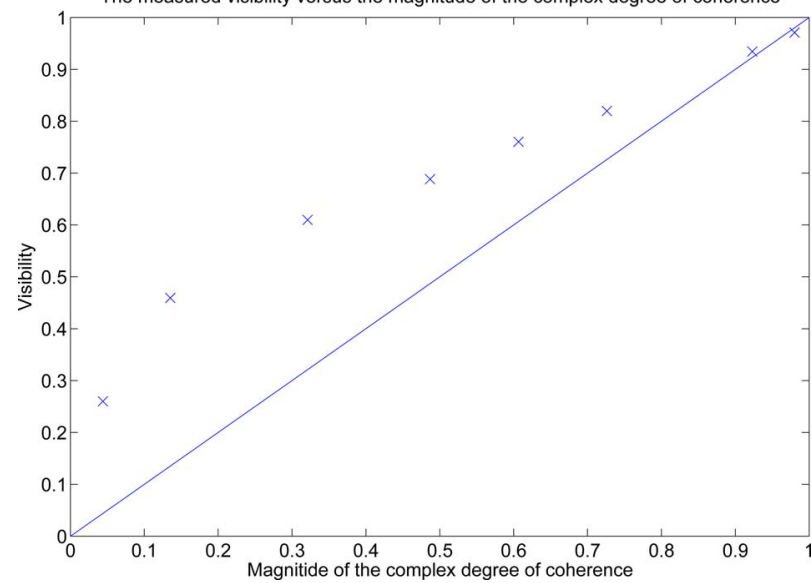

Fig. 9. (Color online) Plot of the visibility versus the magnitude of the complex degree of coherence for a source characterized by $T_{0}=200 \mathrm{fs}, T=50 \mathrm{fs}, \tau_{c}=100 \mathrm{fs}, \sigma_{s}=2 \mathrm{~mm}$ and $\sigma_{g}=1 \mathrm{~mm}$. Each ' $\times$ ' denotes a simulation run with 8000 pulses. The interferometer is simulated to be $2 \mathrm{~mm}$ away from the secondary source (one beam width). The straight line represents a perfect match between the simulated visibility and the calculated magnitude of the complex degree of coherence.

the two source points nearest each pinhole. Each " $\times$ " denotes a different simulation run. In the example considered here $\gamma_{12}\left(\mathbf{r}_{1}, \mathbf{r}_{2}\right)=\exp \left(-\left|\mathbf{r}_{1}-\mathbf{r}_{2}\right|^{2} / 2 \sigma_{g}^{2}\right)=\exp \left(-s^{2} / 2 \sigma_{g}^{2}\right)$. Simulations were run for varying pinhole spacings, $s$, and the measured visibility was plotted against $\gamma_{12}$. When the interferometer is close to the source, the measurements are in reasonable agreement. For pairs of points that are farther from the point of maximal intensity of the beam (points that result in fields that exhibit lower visibility), the approximation that the primary contribution to the field in the pinhole comes from the point in the source directly across from it becomes less valid. This is because the field amplitude at that point may be much smaller than at neighboring points. This explains why the visibility falls off more slowly in Fig. 9 than the magnitude of the complex degree of coherence of the field between the two points nearest the pinholes.

There are, in the literature, several works in which the preceding analysis may be applied [12,14-18]. In one of the experiments described in [16,17], a Young's-style interferometer is used to make visibility measurements of pulsed fields generated by high harmonic generation, with the interferometer a distance of $4 \mathrm{~cm}$ away from the source. This experiment is carried out in the Fresnel zone of the source. The resulting interferograms have visibilities in the range $0.3 \leqslant \mathcal{V} \leqslant 0.6$. Based on the near zone analysis, it is not clear that these visibility measurements say anything about correlations in the near zone of the source. The experiment in [14] uses a waveguide of radius $75 \mu \mathrm{m}$ as a source to illuminate pinholes $60 \mathrm{~cm}$ away, with light of wavelength $13 \mathrm{~nm}$. In this case, the terms appearing on either side of the extreme inequality in inequality (19) are of the same order $\left(z_{0} / a=8 \times 10^{3}, a / 4 \lambda\right.$ $\left.=5.8 \times 10^{3}\right)$, so the measurements are again in the Fresnel zone of the source. The interferogram in Fig. 1 of Ref. [14] suggests that the source is highly spatially coherent, as the authors conclude, though some care should be taken in this interpretation; Fig. 9 shows that the apparent vis- ibility for experiments with pulsed fields consistently overestimates the degree of spatial coherence of the source. The Fresnel zone visibility may be larger or smaller than the visibility in the near zone of the source, depending on which of the three regimes these experiments fall into. The measurements in all of these experiments do indicate that the field at the detector is reasonably partially coherent, but that cannot be generalized back to statements about the source. In the experiments discussed here, a key parameter that differentiates these regimes is unknown, namely $\tau_{c}$, the coherence time. Moreover, in the works mentioned above [12,14-18], the authors do not specify the pulse duration, although this may be determined from far zone measurements [7]. The assumption of stationarity, which is implicit in cw experiments, restricts the way in which the statistics of the problem may be manifest in the outcome of the experiments to a degree only appreciated when this assumption is relaxed. When stationarity is relaxed to cyclostationarity, or relaxed further to general nonstationarity, the outcome of experiments carried out with the traditional interferometers may be connected to the statistical properties of the source or the field only when many of the appropriate parameters are specified. For instance, $T_{0}, T$, and $\tau_{c}$ should all be specified for the cyclostationary case in order to draw the connection between the outcome of the two-pinhole experiment and the spatial coherence properties of the field.

\section{B. Single-Shot Measurements}

The fields presented in this paper differ from the standard stationary theory in that there exists an "on" and "off" time for the radiated field. Depending on the repetition rate of the pulses and the type of detector used, this creates a window in which to collect measurements from each pulse individually. However, these single-shot measurements of intensity $[12,18]$ are not necessarily indicative of the time-averaged intensity measurements as presented in previous sections. Only in certain limiting cases, such as when the pulse time is long compared to the coherence time and the coherence length is long compared with the beam width, might a single-shot measurement be indicative of the time-averaged behavior. In Fig. 10, the interferograms generated by a single pulse (of time $T$ ), averaged over the repetition period $T_{0}$ [i.e., the intensity at a given point is $\bar{I}(P)=\int_{t_{i}}^{t_{i}+T_{0}} \mathrm{~d} t \Gamma(P, P, t, t)$, where $t_{i}$ is the time that the pulse reaches the detector], are shown as would be measured in the Fresnel zone for a field representative of Regime II. The time-averaged measurement over 800 pulses for the same source is shown in Fig. 11. Note that the single-shot measurements of the intensity do not resemble the time-averaged intensity, even though the top panel of Fig. 10 does resemble a traditional interferogram. Only through observing the shot-toshot behavior of the interferograms can one determine whether a single shot is sufficient to characterize the entire pulse train. The width of the peak in the interferograms for the single-shot measurements are indicative of the pulse time, $T$ (an interferogram extending out to $\pm 1.5 \mathrm{~mm}$ corresponds to a pulse time of $200 \mathrm{fs}$ for this geometry) in Regimes I and II. The width of the peak in the time-averaged measurement, though, is indicative of the 


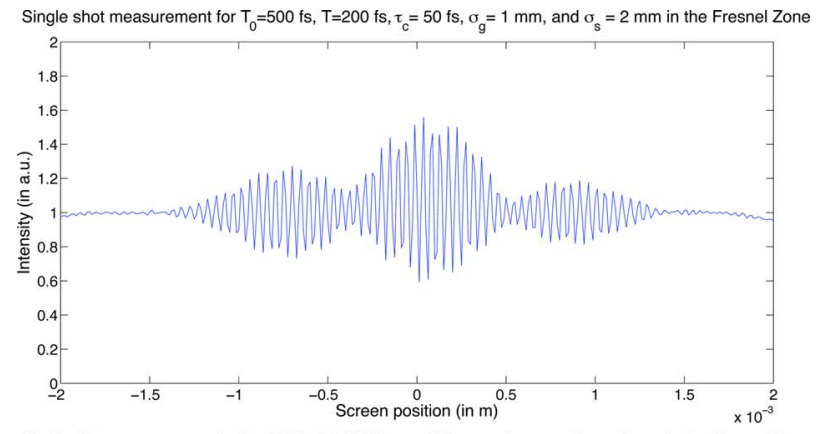

Single shot measurement for $T_{0}=500 \mathrm{fs}, T=200 \mathrm{fs}, \tau_{c}=50 \mathrm{fs}, \sigma_{\mathrm{g}}=1 \mathrm{~mm}$, and $\sigma_{\mathrm{s}}=2 \mathrm{~mm}$ in the Fresnel Zone

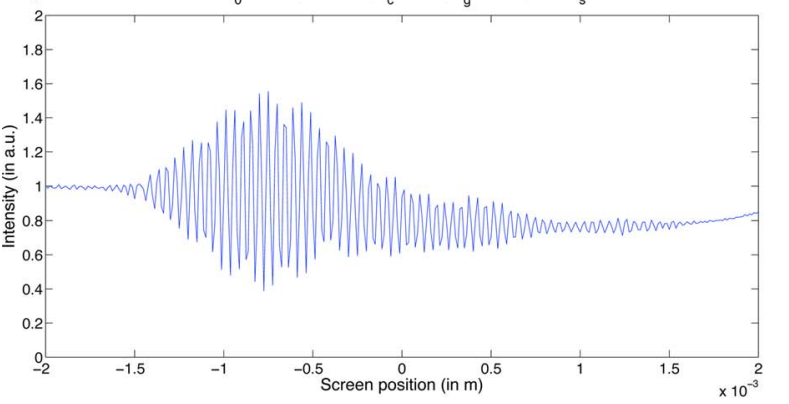

Single shot measurement for $\mathrm{T}_{0}=500 \mathrm{fs}, \mathrm{T}=200 \mathrm{fs}, \tau_{\mathrm{c}}=50 \mathrm{fs}, \sigma_{\mathrm{g}}=1 \mathrm{~mm}$, and $\sigma_{\mathrm{s}}=2 \mathrm{~mm}$ in the Fresnel Zone

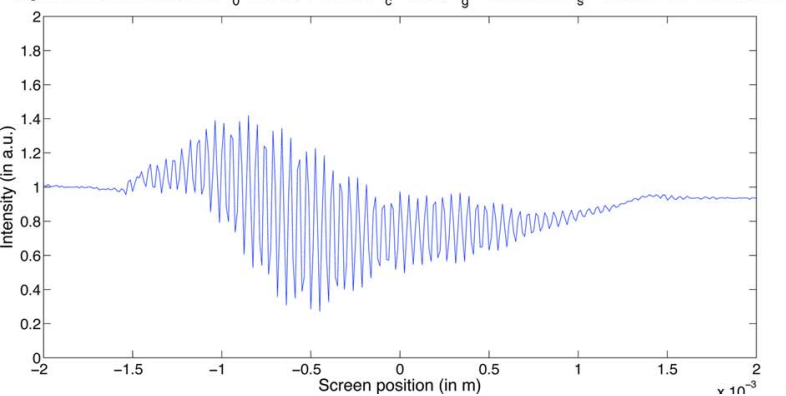

Fig. 10. (Color online) Results of three simulations of a singleshot measurement for a field parameterized by $T_{0}=500 \mathrm{fs}, T$ $=200 \mathrm{fs}, \tau_{c}=50 \mathrm{fs}, \sigma_{s}=2 \mathrm{~mm}$ and $\sigma_{g}=1 \mathrm{~mm}$ as would be measured in the Fresnel zone.

coherence time at the two pinholes (an interferogram extending out to $\pm 0.5 \mathrm{~mm}$ corresponds to a an effective time of $66 \mathrm{fs}$ for this geometry) in Regimes II and III. The coherence time of the field at the pinholes is not the coherence time of the field at the source because of propagation effects.

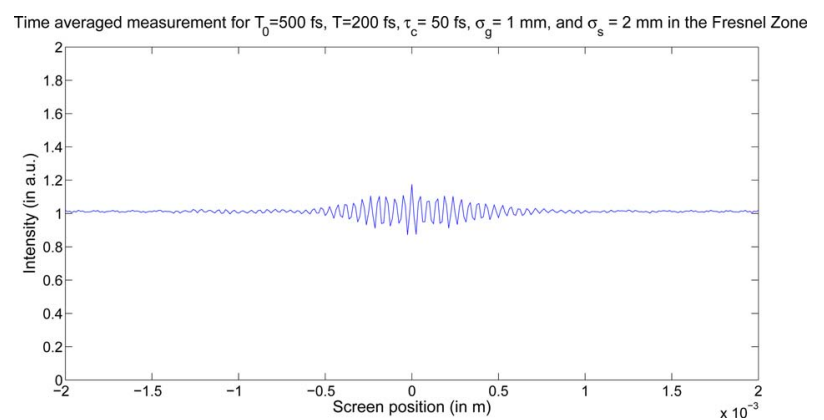

Fig. 11. (Color online) Results of a simulation of the timeaveraged intensity (taken over 800 pulses) for a field parameterized by $T_{0}=500 \mathrm{fs}, T=200 \mathrm{fs}, \tau_{c}=50 \mathrm{fs}, \sigma_{s}=2 \mathrm{~mm}$ and $\sigma_{g}=1 \mathrm{~mm}$ as would be measured in the Fresnel zone.

\section{CONCLUSION}

Using a cyclostationary model, diffraction and interference effects were illustrated for spatially partially coherent ultrafast pulses. It was shown that single-shot measurements of intensity in an interferometer are not necessarily indicative of time-averaged measurements, and thus single-shot measurements cannot necessarily be used to infer coherence properties of the source. Interferometric data were also analyzed for different regions away from the source. The field in the far zone results from equally weighted contributions from all points in the source plane. The spatial coherence of the source thus affects the coherence function in the far zone only through a constant multiplier. In the Fresnel zone, the field results from unequally weighted contributions from multiple points in the source plane, with each contribution associated with a different propagation time. In stationary fields, the statistics are sensitive only to delay differences, whereas in the case considered here, there is a dependence on absolute time that complicates the results considerably. In the near zone, the Young's interferometer essentially samples the field at the source plane at points closest to the pinholes. As a result, the effects of partial spatial coherence of the source are manifested as a constant multiplier on the interference terms, and the temporal coherence determines unambiguously the shape of the interferogram. Based on this analysis, it was also shown that from measurements in the far zone, temporal properties of the source, i.e., the minimum of the coherence time or pulse length, can be inferred and that the spatial coherence length of the source can be determined only from measurements in the near zone.

\section{ACKNOWLEDGMENTS}

R. W. Schoonover, B. J. Davis, and P. Scott Carney acknowledge support by the National Science Foundation (NSF) under CAREER Grant No.0239265 and the U. S. Air Force Office of Scientific Research (USAFOSR) under Grant No. MURI F-49620-03-1-0379. R. A. Bartels acknowledges support from the National Science Foundation (NSF) under Award ECS-0348068 and support from a Sloan Research Fellowship.

\section{REFERENCES}

1. L. Mandel and E. Wolf, Optical Coherence and Quantum Optics (Cambridge Univ. Press, 1995).

2. E. Wolf, Introduction to the Theory of Coherence and Polarization (Cambridge Univ. Press, 2007).

3. N. Wiener, "Generalized harmonic analysis," Acta Math. 55, 117-258 (1930).

4. A. Khintchine, "Korrelationstheorie der stationären stochastischen Prozesse," Math. Ann. 109, 604-615 (1934).

5. A. Einstein, "Méthode pour la détermination de valeurs statistiques d'observation concernant des grandeurs soumises à des fluctuations irrégulières," Arch. Sci. Phys. Nat. 37, 254-256 (1914).

6. H. Lajunen, J. Tervo, J. Turunen, P. Vahimaa, and F. Wyrowski, "Spectral coherence properties of temporally modulated stationary light sources," Opt. Express 11, 1894-1899 (2003).

7. R. Schoonover, B. Davis, R. Bartels, and P. Carney, "Optical interferometry with pulsed fields," J. Mod. Opt. 55, 1541-1556 (2008) 
8. I. G. Fuss, "An interpretation of the spectral measurement of optical pulse-train noise," IEEE J. Quantum Electron. 30, 2707-2710 (1994).

9. D. J. Jones, S. A. Diddams, J. K. Ranka, A. Stentz, R. S. Windeler, J. L. Hall, and S. T. Cundiff, "Carrier-envelope phase control of femtosecond mode-locked lasers and direct optical frequency synthesis," Science 288, 635-639 (2000).

10. T. Udem, R. Holzwarth, and T. Haensch, "Optical frequency metrology," Nature 416, 233-237 (2002).

11. V. Devrelis, M. O'Connor, and J. Munch, "Coherence length of single laser pulses as measured by CCD interferometry," Appl. Opt. 34, 5386-5389 (1995).

12. V. Papadakis, A. Stassinopoulos, D. Anglos, S. H. Anastasiadis, E. P. Giannelis, and D. G. Papazoglou, "Single-shot temporal coherence measurements of random lasing media," J. Opt. Soc. Am. B 24, 31-36 (2007).

13. R. Bartels, A. Paul, H. Green, H. Kapteyn, M. Murnane, S. Backus, I. Christov, Y. Liu, D. Attwood, and C. Jacobsen, "Fully spatially coherent EUV beams generated using a small-scale laser," Science 297, 376-378 (2002).

14. X. Zhang, A. Libertun, A. Paul, E. Gagnon, S. Backus, I. Christov, M. Murnane, H. Kapteyn, R. Bartels, Y. Liu, and D. T. Attwood, "Highly coherent light at $13 \mathrm{~nm}$ generated by use of quasi-phase-matched high-harmonic generation," Opt. Lett. 29, 1357-1359 (2004).

15. L. Le Déroff, P. Salières, B. Carré, D. Joyeux, and D. Phalippou, "Measurement of the degree of spatial coherence of high-order harmonics using a Fresnel-mirror interferometer," Phys. Rev. A 61, 35 (2000).

16. T. Ditmire, E. Gumbrell, R. Smith, J. Tisch, D. Meyerhofer, and M. Hutchinson, "Spatial coherence measurement of soft $\mathrm{x}$-ray radiation produced by high order harmonic generation," Phys. Rev. Lett. 77, 4756-4759 (1996).

17. T. Ditmire, J. Tisch, E. Gumbrell, R. Smith, D. Meyerhofer, and M. Hutchinson, "Spatial coherence of short wavelength high-order harmonics," Appl. Phys. B: Photophys. Laser Chem. 65, 313-328 (1997).

18. Y. Nagata, K. Furusawa, Y. Nabekawa, and K. Midorikawa, "Single-shot spatial-coherence measurement of $13 \mathrm{~nm}$ highorder harmonic beam by a Young's double-slit measurement," Opt. Lett. 32, 722-724 (2007).

19. D. Eliyahu, R. A. Salvatore, and A. Yariv, "Noise characterization of a pulse train generated by actively mode-locked lasers," J. Opt. Soc. Am. B 13, 1619-1626 (1996).

20. H. Lajunen, J. Tervo, and P. Vahimaa, "Overall coherence and coherent-mode expansion of spectrally partially coherent plane-wave pulses,” J. Opt. Soc. Am. A 21, 2117-2123 (2004).

21. R. Schoonover, B. Davis, and P. Carney, "The generalized Wolf shift for cyclostationary fields," Opt. Express 17, 4705-4711 (2009).

22. W. Gardner, A. Napolitano, and L. Paura, "Cyclostationarity: half a century of research," Signal Process. 86, 639-697 (2006).

23. A. Yaglom, Correlation Theory of Stationary and Related Random Functions (Springer, 1987).

24. B. Davis, "Measurable coherence theory for statistically periodic fields," Phys. Rev. A 76, 043843 (2007).

25. J. Eberly and K. Wodkiewicz, "The time-dependent physical spectrum of light," J. Opt. Soc. Am. 67, 1252-1261 (1977).

26. M. Lewenstein, P. Balcou, M. Y. Ivanov, A. L'Huillier, and P. B. Corkum, "Theory of high-harmonic generation by low-frequency laser fields," Phys. Rev. A 49, 2117-2132 (1994).

27. J. Degnan, N. Center, and M. Greenbelt, "Theory of the optimally coupled Q-switched laser," IEEE J. Quantum Electron. 25, 214-220 (1989).

28. V. Torres-Company, H. Lajunen, and A. Friberg, "Coherence theory of noise in ultrashort-pulse trains," J. Opt. Soc. Am. B 24, 1441-1450 (2007).

29. V. Torres-Company, H. Lajunen, and A. Friberg, "Effects of partial coherence on frequency combs," J. Eur. Opt. Soc. Rapid Pub. 2 (2007).

30. T. Young, "The Bakerian lecture. Experiments and calculations relative to physical optics," Philos. Trans. R. Soc. London 94 (1804).

31. T. Jannson, ed., Tribute to Emil Wolf: Science and Engineering Legacy of Physical Optics (SPIE Press, 2004).

32. M. Born and E. Wolf, Principles of Optics: Electromagnetic Theory of Propagation, Interference and Diffraction of Light, 7th (expanded) ed. (Cambridge Univ. Press, 1999).

33. B. Davis, "Simulation of vector fields with arbitrary secondorder correlations," Opt. Express 15, 2837-2846 (2007). 